\title{
Breaking the Ice in Human-Agent Communication: Eye-Gaze Based Initiation of Contact with an Embodied Conversational Agent
}

\author{
Nikolaus Bee, Elisabeth André, and Susanne Tober \\ Institute of Computer Science, Augsburg University, \\ 86135 Augsburg, Germany \\ \{bee, andre\}@informatik. uni-augsburg.de
}

\begin{abstract}
In human-human conversation, the first impression decides whether two people feel attracted by each other and whether contact between them will be continued or not. Starting from psychological work on flirting, we implemented an eye-gaze based model of interaction to investigate whether flirting tactics help improve first encounters between a human and an agent. Unlike earlier work, we concentrate on a very early phase of human-agent conversation (the initiation of contact) and investigate which non-verbal signals an agent should convey in order to create a favourable atmosphere for subsequent interactions and increase the user's willingness to engage in an interaction with the agent. To validate our approach, we created a scenario with a realistic 3D agent called Alfred that seeks contact with a human user. Depending on whether the user signals interest in the agent by means of his or her gaze, the agent will finally engage in a conversation or not.
\end{abstract}

\section{Introduction}

In human-human conversation, the first impression decides whether two people feel attracted by each other and whether contact between them will be continued or not. A large industry has developed around the production of training material that intends to give advice to people that wish to present themselves in a positive light to new people (for example, see [Cohen, 1992, for a guidebook on flirting). A recommendation that can be found in almost any guidebook that prepares people for such first encounters, whether be it in a date or a job interview, is to show a genuine interest in the conversational partner using eye gaze and smiles. In this way, instant rapport is built up which creates a good starting point for subsequent interactions (see Argyle and Cook, 1976 and Kleinke, 1986]).

Recently, there has been a significant amount of work on embodied conversational agents that make use of non-verbal behaviors to establish rapport with a human user. There is empirical evidence that rapport-building tactics also work for human-agent communication, see, for example, Gratch et al., 2006]. Most work focuses on the use of non-verbal signals during a dialogue taking it for 
granted that the human has an interest in communicating with the agent. Unlike earlier work, our paper concentrates on a very early phase of human-agent communication (the initiation of contact) and investigate which non-verbal signals an agent should convey in order to create a favourable atmosphere for subsequent interactions and increase the user's willingness to engage in an interaction with the agent. In particular, we are interested in the question of whether it is possible to give a human user the feeling that an agent has a genuine interest in him or her. We consider work on flirt tactics as a useful resource to implement agents that have these capabilities.

To validate our approach, we created a scenario with a realistic 3D agent called Alfred that seeks contact with a human user. Depending on whether the user signals interest in the agent by means of his or her gaze, the agent will finally engage in a conversation or not. Alfred is able to convey emotional and attentive states via facial displays, head movements and gaze shifts. In addition, we employ a contact-free eye tracker that provides us continuously with information on the user's eye gaze. In order to use means to analyze and generate social signals effectively, the agent has to sense the user's eye gaze and to align it in parallel with its own behaviors. The challenge of our work is to tightly synchronize the agent's non-verbal behaviors with the user's non-verbal feedback.

\section{Related Work}

So far, embodied conversational agents have rarely been used as flirt partners. An exception includes the work by Pan and Slater, 2007 who present a virtual party-like environment in which a female character called Christine approaches a male user and involves him into a conversation. The character shows her interest into the male user by smiles and head nods. She leans her upper body forward towards the user, looks at him and maintains eye contact with him. After some time, the character moves closer and formulates personal questions and statements. Using physiological measurements, Pan and Slater, 2007 found out that the participants' level of arousal was correlated to compliments and intimate questions of the character. In addition, some of them indicated in a questionnaire that they had the feeling to have flirted with a real woman.

Unlike Pan and Slater, we do not make use of a full-body character, we just show the upper body of the agent in order to make sure that the users are able to perceive the subtle signals in the agent's face. Furthermore, Pan and Slater's agent is not fully responsive since it is not able to recognize the user's eye gaze. Finally, the courtship behaviors of our agent are much more subtle concentrating on eye gaze behaviors and light smiles. We are less interested in the simulation of flirting per se, but rather aim at investigating to what extent courtship behaviors may contribute to the creation of instant rapport.

Gratch et al., 2006 developed a so-called rapport agent which acts as a silent listener. The agent tries to create rapport by providing rapid non-verbal feedback, expressed by head nods, posture shifts and eye gaze behaviors, through 
a shallow real-time analysis of the human's voice, head motion and body posture. An empirical study revealed that the agent increased speaker fluency and engagement.

The robotic penguin developed by Sidner et al., 2004 is able to track the face of the conversational partner and adjusts its gaze towards him or her. Even though the set of communicative gestures was strongly limited, an empirical study revealed that users indeed seem to be sensitive to a robots conversational gestures and establish mutual gaze with it.

While most research focuses on how to create rapport in short-term interactions, Cassell and Bickmore, 2003 investigate how to establish and maintain a relationship between a user and an agent over a series of conversations. They performed a series of experiment with a virtual character acting as a real-estate agent which revealed that the character's use of social language had an important impact on the creation of rapport.

Most studies analyzing the user's non-verbal feedback behavior make use of head trackers. They are able to roughly assess in which direction the user is looking, but do not have more detailed information on the user's eye gaze direction. One of the earliest work of using eye trackers for agent-based human interaction comes from Starker and Bolt, 1990. They adapt "The Little Prince" to the users current interest in a virtual scene that shows one planet from the story by Antoine de Saint-Exupéry. Dependent on the duration and focus of the user's gaze further details of the scene are described via a text-to-speech system. Another exception includes the work by Eichner et al., 2007] who made use of an eye tracker. In an experiment, they showed that agents that adapted the content of their presentation to a user's eye gaze were perceived as more natural and responsive than agents that did not have that capability.

The studies above show that embodied conversational agents are to a certain extent able to establish rapport with human conversational partners through appropriate verbal and non-verbal behaviors. Unlike the approaches described above, we focus on the first seconds of an encounter and investigate how to create a friendly and natural atmosphere for human-agent communication by appropriate courtship behaviors of the agent. We make use of an eye tracker to monitor the user's gaze behaviors. A particular challenge of our work is to align and synchronize the user's gaze behaviors with the agent's courtship behaviors.

\section{Eye Gaze Model for Human-Agent Interaction}

As a basis for our research, we rely on the approach by Givens, 1978 who distinguishes between five phases of flirting:

- The attention phase describes the phase in which men and women arise each other's attention. It is characterized by ambivalent non-verbal behavior, such as a brief period of mutual gaze broken by downward eye aversion, reflecting the uncertainty of the first seconds.

- In the recognition phase, one interactant recognizes the interest of the other. He or she may then disencourage the other interactant, for example, by a 
downward gaze, or signal readiness to continue the interaction, for example, by a friendly smile.

- After mutual interest has been established, the man or woman may be initiated the interaction phase and engage in a conversation.

- In the sexual-arousal and resolution phases, the relationship between man and woman intensifies. These two phases are not further described here because of their missing relevance to human-agent communication.

Our system will cover the attention, recognition and the initiation of the interaction phase. In addition to the work by Givens, our eye gaze based interaction system incorporates findings from Tramitz, 1992 and Bossi, 1995. In particular, we rely on their work to determine the timing of gazes. Tramitz, 1992 analyzed the flirt behavior in a study with 160 school students. She found that the initiation of a first encounter decides on the continuation of the flirt interaction. Bossi, 1995 used the same study but analyzed couples with different levels of interest on each other. Flirting couples seemed to use more time, up to three times, to gaze at each other. Further, the first gaze and following single gazes lasted longer.

Attention Phase. The implementation of the attention phase (see Fig. 1 left) is motivated by typical behavior sequences described in Givens, 1978. The attention phase starts at the point when the human and the virtual agent take notice of each other. The virtual agent shows a slightly friendly facial expression and the gaze is averted from the user. First, the agent's eyes only wander around
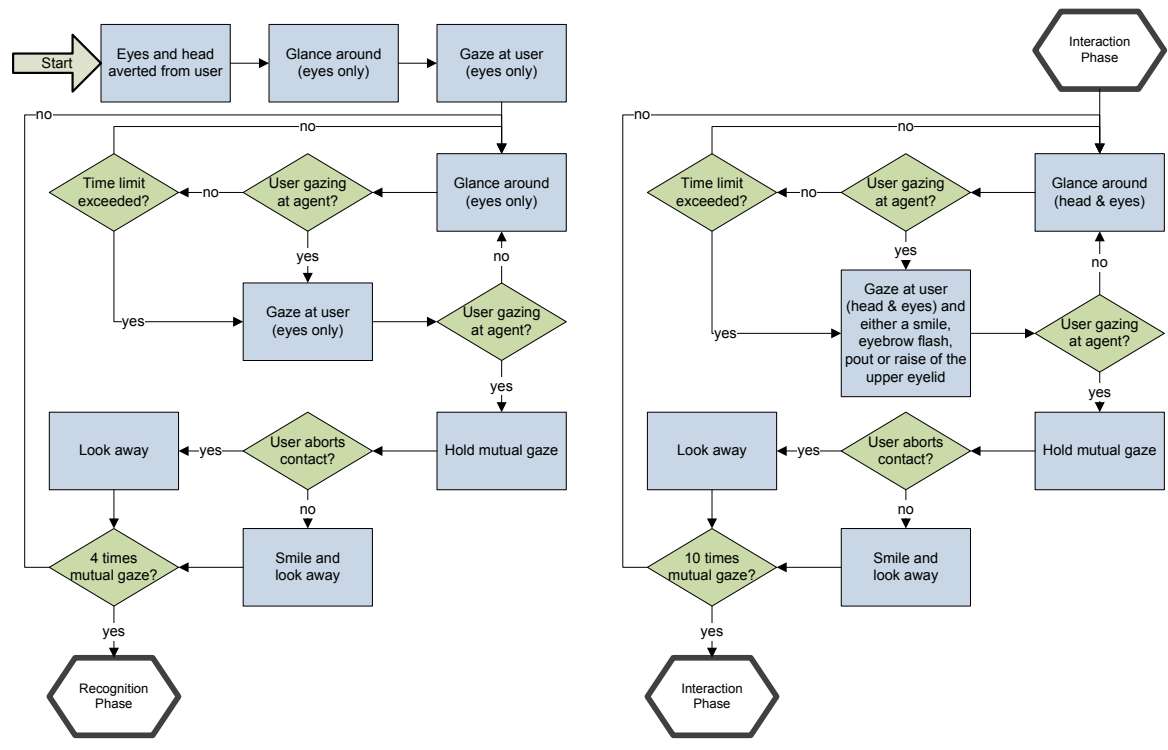

Fig. 1. Attention phase (left) and recognition phase (right) 
the room for a while until they meet the user's eyes. After that, the virtual agent will engage in an interplay of mutual and averted gaze.

While the virtual agent gazes randomly around the room, the system checks whether the user gazes at the agent. If this is the case, the agent establishes gaze contact with the user. The system is now in the hold-mutual-gaze-state and the agent tries to hold eye contact until a specified time interval elapses. It then breaks off eye contact by a downward gaze accompanied by a smile. To avoid that unconscious very brief sweep gaze behaviors of the user are by mistake categorized as mutual gaze, eye contact with the user will be taken into account only after a certain duration. In case the user breaks off eye contact before the maximal time interval elapses, the agent will look away as well, however, without showing a smile since the user would not recognize the facial expression anymore. If the user does not respond to eye contact established by the agent, the agent will avert its gaze again. After each successful or failed eye contact, the system will return to the state of looking around trying to establish eye contact again or to respond to the user's eye gaze.

This loop is repeated until one of the following terminating conditions is fulfilled. In the positive case, a certain number of mutual gazes could be established and the system transits to the next phase. In the negative case, the agent has attempted to established gaze contact with user in vain and breaks off the complete interaction due to missing interest of the human flirt partner. After each successful gaze contact, the emotional state of the agent improves and its facial display becomes more joyful. After each failed attempt to establish gaze contact, the emotional state of the agent becomes worse and it looks more sad.

Recognition Phase. Similar to the attention, the recognition phase (see Fig. 1 right) is based on the interplay between mutual and averted gaze behavior, save that the durations of the mutual gazes increase. Further, the virtual agent smiles more often and uses more distinct flirt signals (i.e. eyebrow flash, pout or raise of the upper eyelid). Since the agent's self confidence has increased after the successful attention phase, it tries to establish eye contact more often and eye movements are supported by head movements to show a more obvious interest in the human interactant. Just as the attention phase, the recognition phase can still fail. Namely, in case the agent unsucessfully tried to establish mutual gaze for several times. Or, if a particular number of mutual gazes has been set up, the recognition phase was successful and completed. This will lead to the next phase, which is the interaction phase.

Interaction Phase. After successfully completing the two previous phases, verbal communication will be initiated in the interaction phase. The virtual agent verbally addresses the user using small talk strategies adopted from Tramitz, 1992.

\section{System}

The system for eye gaze based interaction between the virtual agent and human consists of an eye tracker, an virtual character and the program logic for the 
interaction. We use a contact-free eye tracker (SMI iView X RED) which allows the user to move relatively free.

\subsection{Eye Tracker}

The SMI iView X RED eye tracker operates with sampling rate of $50 \mathrm{~Hz}$, the latency for a gaze point is less than $35 \mathrm{~ms}$ and the tracking accuracy is less than $0.5^{\circ}$. The distance between the eye tracker and the user should be about 60 $80 \mathrm{~cm}$ [SMI, 2008. The advantages of an unobtrusive, contact-less eye tracker include that users do not have to wear a sometimes bulky apparatus and thus are not steadily reminded that their gaze is tracked. Further, the SMI iView X RED eye tracker allows head movements horizontally and vertically up to $20 \mathrm{~cm}$ in each direction.

\subsection{Virtual Agent}

We used a fully controllable virtual head which was developed by Augsburg University and which is freely available Augsburg University, 2008]. The head is based on Ekman and Friesen's Facial Action Coding System (FACS). FACS was developed to classify human facial expressions Ekman and Friesen, 1975. It divides the face into action units (AU) to describe the different expressions a face can display (e.g. inner brow raiser, nose wrinkler, or cheek puffer). Although FACS was originally designed to analyze natural facial expressions, it turned out to be usable as a standard for production purposes, too. That is why FACS based coding systems are used with the generation of facial expressions displayed by virtual characters, like Kong in Peter Jackson's King Kong. But the usage of FACS is not only limited to virtual characters in movies. The gaming industry with Half-Life 2 by Valve also utilizes the FACS system to produce the facial expressions of their characters.

Alfred (see Fig. 2), a butler-like character, uses the FACS to synthesize an unlimited set of different facial expressions. The action units were designed using morph targets and thus gives the designer the full power in defining the facial expression outlook. The system includes a tool to control the single action units Bee et al., 2009. The tool allows to store the result in a XML file for later usage in our agent system.

Alfred's mesh has a resolution of about 21.000 triangles. For displaying more detailed wrinkles in the face, normal maps baked from a high-resolution mesh are used. The morph targets for the action units are modeled using the actor's templates from the Facial Expression Repertoire, 2008 (FER).

\subsection{System for Interaction}

To be able to detect where the user is looking at, we connected the eye tracker with the virtual 3D-world. Ray casting allows us to map the screen coordinates obtained from the eye tracker to the objects in the virtual world. In this vein, we are able to detect whether the user looks at the virtual agent, the left eye or 


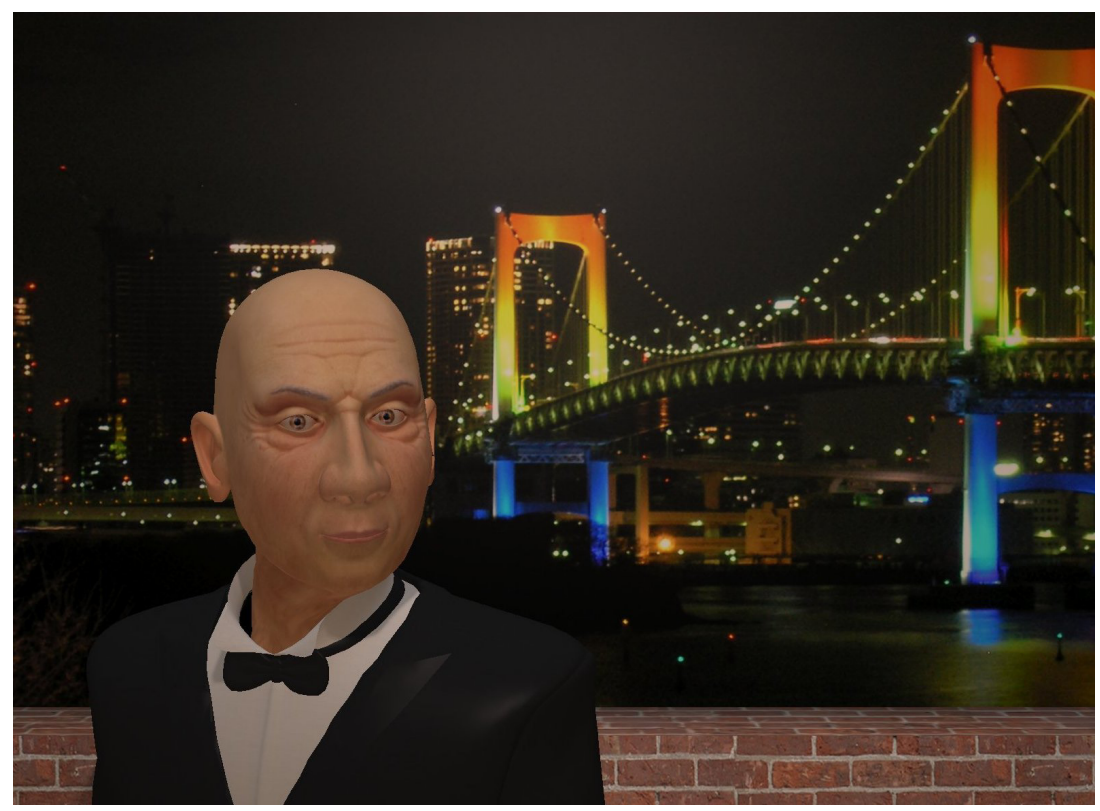

Fig. 2. The virtual character Alfred with a skyline in the background

the right eye or something else in the virtual scene. This was necessary for the eye gaze based interaction on a level of mutual gaze and to see, if the user is looking at Alfred or not.

The flirt behavior can be varied by parameters. A confidence value defines the agent's level of self assurance and influences the probability that the agent initiates gaze interaction. The maximal and minimal duration of mutual gaze can be set as well. Furthermore, we may indicate the maximal duration the virtual agent gazes around. For the attention and recognition phases, the maximum number of trials to initiate mutual gaze before it fails can be defined. Finally, we may specify how long the virtual agent waits until the user responds with mutual gaze. These parameters are stored in a XML file and can be easily adjusted.

The virtual agent is able to direct his gaze using his eyes only or his head and his eyes in combination.

Flirt signals are displayed whenever mutual gaze occurs. In the attention phase, flirt signals are rarely sent whereas in the recognition phase, flirt signals are an integral part of the interaction. Whenever mutual gaze occurs, the virtual agent sends with a probability of $1 / 3$ one of the following flirt signals: an eyebrow flash, a pout, a raise of the upper eyelid or a smile.

The agent's mood changes dependent on the number of successful mutual gazes. The more mutual gazes occur, the friendlier Alfred's facial expression becomes. Vice versa, the agent's happiness declines if there is no reaction from the user to an attempt to establish mutual gaze. 


\section{Evaluation}

We conducted an empirical study to demonstrate the benefits of eye gaze based interaction in combination with flirting tactics. Our main focus was to figure out if the users realize the virtual agent's interest. Further, we would like to investigate the impact of an eye gaze based interaction system on user engagement. Finally, we were interesting in finding out whether an eye gaze based interaction system works with a life-size setting. Thus, the study focuses on the following points:

1. The flirting agent is able to show the user that it has an interest in him through its gaze and facial expression behavior, and the user will perceive this behavior as flirting.

2. The integration of flirting tactics has a positive impact on the perception of the agent and the interaction with him and thus contributes to the user's engagement.

3. By tracking the user's eye gaze and responding to it in real-time, the effects can be increased.

\subsection{Setting}

The optimal dimensions for such a video projector based eye tracking setting are limited. The user is placed in front of a table on which the eye tracker was placed. The eye tracker with an incline of $23^{\circ}$ is placed $80 \mathrm{~cm}$ above ground and $140 \mathrm{~cm}$ away from the projection surface. The user is seated $60-80 \mathrm{~cm}$ in front of the eye tracker. In total the user is about $2 \mathrm{~m}$ away from the virtual agent, which is within the social space according to Hall, 1963. The projection surface sizes $120 \times 90 \mathrm{~cm}$, which displays the virtual agent in life-size (see Fig. 3).

To avoid that the user automatically stares at the virtual agent (which would happen if it was placed in the center of the visual display), we placed it on the left side. To offer an enriched scene where the user has the choice to look away from the virtual agent, we added a city skyline (see Fig. 2).

Interaction Modes. Apart from the fully interactive version where the agent recognizes and responds to the user's eye gaze behavior in real-time, two further gaze behavior variants were created to demonstrate the benefits of the gaze behavior model described in Section 3, a non-interactive version which implements an ideal flirt behavior derived from the literature and a non-interactive version which implements an anti-flirt behavior.

In the non-interactive ideal version the virtual agent behaves like in the interactive version except for that it does not respond to the user's eye gaze behavior, but assumes a perfect eye gaze behavior from the user and thus follows a fixed sequence. The gaze directions while glancing around the scene are still randomly selected, but the virtual agent gazes at the user always with the same duration, no matter whether the user returns the gaze or not. 


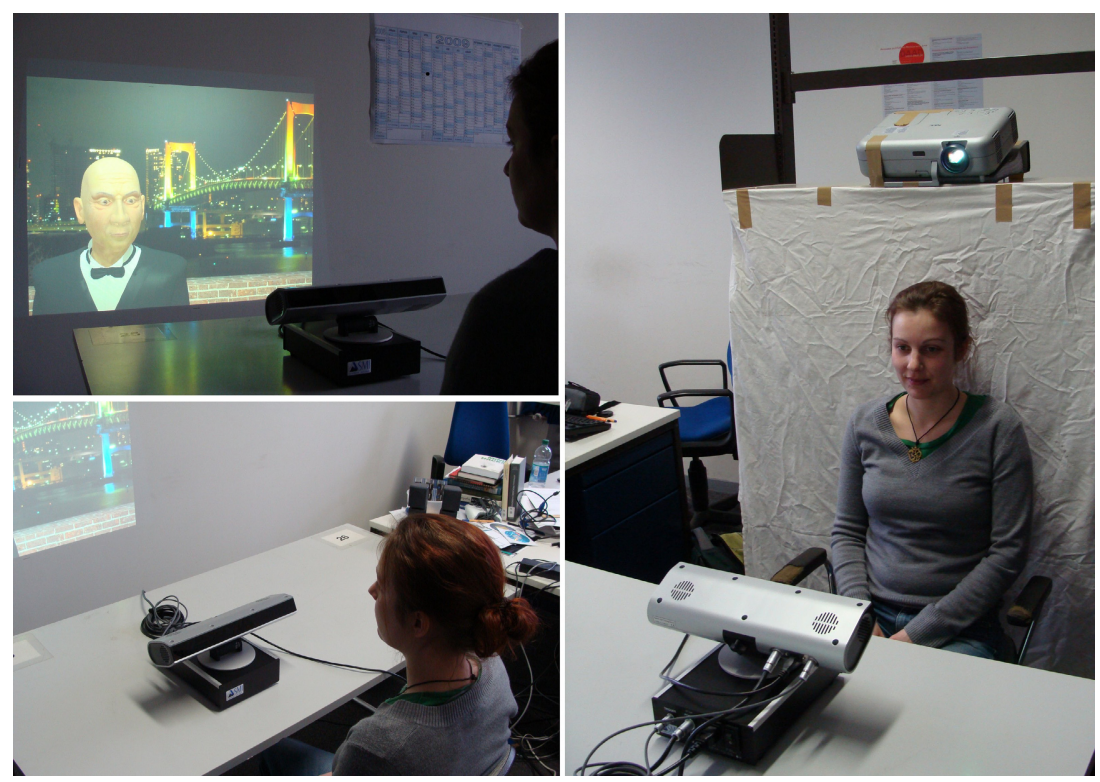

Fig. 3. Set-up for the eye gaze based interaction application from different perspectives

In the non-interactive anti-flirt version, by contrast, the virtual agent behaves contrarily to the typical flirt behaviors previously described. The duration of the mutual gaze is increased from 3 seconds to 7 seconds, which is commonly considered as staring. Furthermore, the facial expression remains neutral, which can be interpreted as a bored attitude towards the user. Finally the virtual agent looks away upwards after gazing at the user instead of downwards.

We had to disable the break after an unsuccessful attention phase in the interactive version as the interaction duration would have been significantly shorter and thus not comparable to the two non-interactive versions.

\subsection{Study}

For the study, we recruited 16 subjects, solely women due to the male virtual counterpart, and presented them with all three interaction modes. The order of the three interaction modes (i.e. interactive, non-interactive ideal and noninteractive anti-flirt) was randomized for each subject to avoid any bias due to ordering effects. The procedure was as follows: First, the subjects were asked to fill in the first part of the questionnaire about demographic data. After placing the subjects in front of the eye tracker, a calibration, which took less than 2 minutes, was carried out. The subjects were told that they would be presented with a flirting agent and should try to engage in a flirt with the agent themselves using their eye gaze. They were informed that they would have to run the interaction sequence three times, but they did not know that there were different modes of interaction. After accomplishing one interaction sequence, the subjects 
had to fill in a post-sequence questionnaire about the interaction with the virtual agent. The study took about 20 minutes including the calibration for the eye tracker and answering the questionnaire.

\subsection{Questionnaire}

The post-sequence questionnaire used 13 attitude statements with a 5-point Likert scale to evaluate how the participants perceived the interaction with the system. The questions were related to the user's engagement of the interaction (five questions: E1 - E5), the exclusion of external influences (three questions: I1 - I3) and the quality of the gaze behavior model (five questions: Q1 - Q5).

\subsection{Results}

The analyses of the questionnaires were based on the one-way analysis of variance (ANOVA) across the different groups and the Tukey-HSD for the posthoc twosided pairwise comparisons. Two subjects had to be excluded from the analysis due to technical difficulties with the eye tracker. In their case, the eye tracking data stream was discontinuous and thus the interactive version did not work properly.

Engagement of the Interaction. The one-way ANOVA for the questions regarding the engagement revealed significant differences among the means for question $\mathrm{E} 1\left(F(2,39)=3.02, p<0.05, \eta^{2}=4.02\right), \mathrm{E} 2(F(2,39)=6.07, p<0.01$, $\left.\eta^{2}=6.5\right), \mathrm{E} 3\left(F(2,39)=5.98, p<0.01, \eta^{2}=6.38\right)$ and $\mathrm{E} 5(F(2,39)=3.35$, $\left.p<0.05, \eta^{2}=2.31\right)$. E4 did not reveal significant differences. The Tukey-HSD posthoc test for pairwise comparisons revealed a significant difference for E1 between the interactive and anti-flirt mode $(p<0.05)$, for E2 between the interactive and anti-flirt mode $(p<0.01)$ and for E3 between the ideal and anti-flirt mode $(p<0.05)$ and the interactive and anti-flirt mode $(p<0.01)$.

Questions E1 - E5 (see Fig. 4) were related to the users' engagement in the eye gaze interaction with the virtual agent. All these questions resulted into a higher mean for the interactive mode, where the agent's gaze behavior was aligned to the user's eye gaze. In the interactive mode, the subjects rated Alfred's eye gaze behavior and mimics more realistic (E1) and enjoyed the interactions with Alfred more (E2). Furthermore, they uttered a higher interest in continuing the interaction with Alfred (E3) and in actually engaging in a conversation with him (E4). They also thought that their own interaction behavior was more natural in the interactive mode than in the two non-interactive modes.

Exclusion of External Influences. The one-way ANOVA for the questions (I1 - I3) regarding the exclusion of external influences revealed no significant differences (see Fig. 4). Unaffected by the mode of interaction, the subjects felt hardly watched by the equipment (I1). Furthermore, they gave similar subjective ratings for their flirting capabilities independent from the mode of interaction 


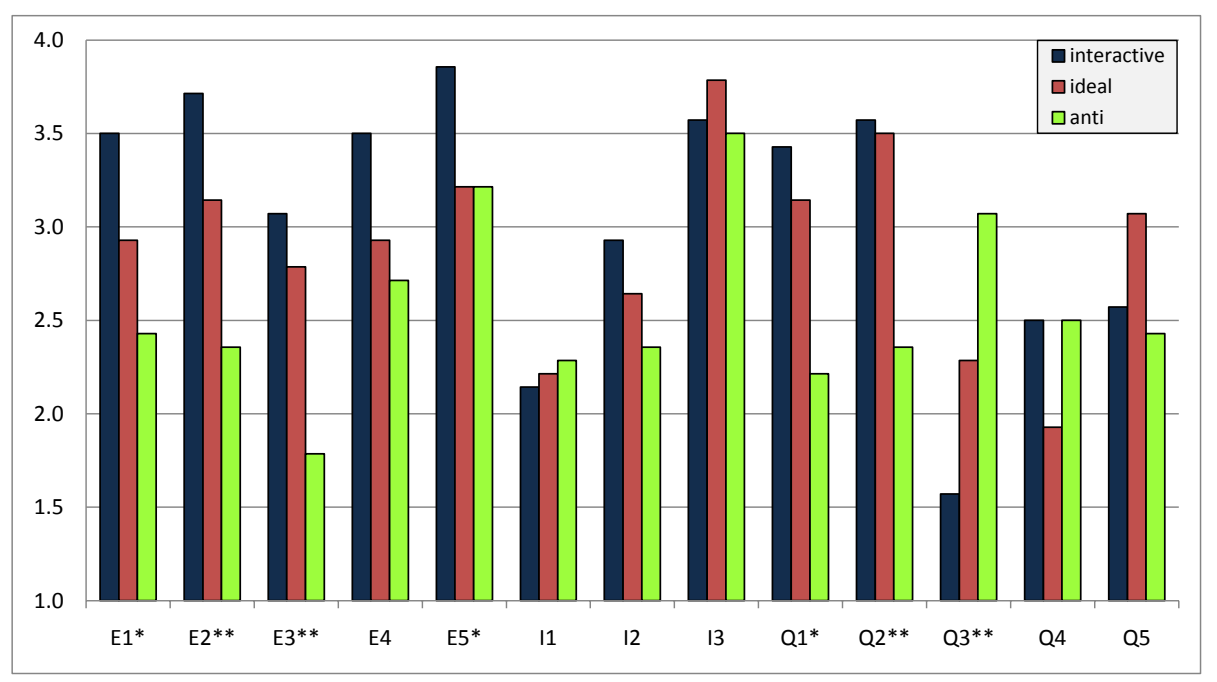

Fig. 4. Results of the questionnaire for the interactive, non-interactive ideal and noninteractive anti-flirt mode $\left({ }^{*} p<0.05,{ }^{* *} p<0.01\right)$

(I2) which we take as further evidence that they did not feel more disturbed in the interactive version. Finally, in all three modes of interaction, the subjects had the feeling that Alfred was looking into their eyes when he was looking at them (I3). Consequently, we can exclude artefacts due to different user sizes and the resulting different positions relative to the agent.

Quality of the Gaze Behavior Model. The one-way ANOVA tests reveal significant differences for all questions regarding the quality of the gaze behavior model, for question Q1 $\left(F(2,39)=4.27, p<0.05, \eta^{2}=5.64\right)$, Q2 $(F(2,39)=$ $\left.5.27, p<0.01, \eta^{2}=6.5\right)$ and $\mathrm{Q} 3\left(F(2,39)=6.00, p<0.01, \eta^{2}=7.88\right)$. The Tukey-HSD posthoc test for pairwise comparisons reveals significant difference for Q1 between the interactive and anti-flirt mode $(p<0.05)$, for Q2 between the ideal and anti-flirt mode $(p<0.05)$ and the interactive and anti-flirt mode $(p<0.05)$ and for Q3 between the interactive and anti-flirt mode $(p<0.01)$.

Questions Q1 and Q2 referred to the ability of the virtual agent to convey interest through its gaze behavior and mimics. The subjects had the impression that Alfred was more interested in them (Q1) and flirting more with them (Q2) in the interactive version than in the other two versions. Nevertheless, they did not have the feeling that Alfred's gaze behaviors were obtrusive (Q3).

Q4 and Q5 did not reveal any significant differences. The subjects felt that the agent gazed at them directly (Q4) and was interactive (Q5) with a medium score in all three conditions. Surprisingly, the user did not perceive the interactive agent as more interactive even though this agent was more positively rated (E1 - E5). 


\subsection{Analyzing the User's Eye Gaze Behavior During the Interaction}

In the following, we perform a more careful analysis of the interactive mode. Here, the overall interaction took between 59.1 and 107.8 seconds, 82.6 seconds on average. The first part of the interaction was completed on average after 24.5 seconds (17.1 seconds minimum and 32.0 seconds maximum) which corresponds to the observation by Tramitz, 1992 that the first 30 seconds of an interaction lay the foundations for further interactions.

As it turned out, the user was more pro-active than the agent in establishing and breaking off eye contact. In $76.9 \%$ of the cases, the gaze contacts were initiated by the human user. In $85.7 \%$ of the cases, the human user decided to break off eye contact.

Four subjects did not execute downward gazes after breaking off eye contact which are typical of flirting situations. Also the remaining candidates showed this behavior only one to three times.

Not every attempt to establish gaze contact also led to mutual gaze. Each subject experienced at least once (at a maximum five times) the situation that the agent did not respond to his or her attempt to establish mutual gaze. As a reason we indicate that the user was averting his gaze immediately after meeting the agent's eyes. That is in some cases, Alfred's response came too late. On the other hand, in 12 out of 14 interactions, it happened only once that the agent tried in vain to establish eye contact.

\subsection{Discussion}

Overall, the experiment led to promising results. In the interactive and the ideal mode, the agent was able to show the users that he had an interest in them and the users also had the feeling that he was flirting with them (Hypothesis I). Furthermore, we found that the effect was increased when moving from the ideal to the interactive mode (Hypothesis II). Although significant differences were only detected between the interactive and the anti-flirt version, the means of the interactive version were always rated higher than the ideal version and the means of the ideal version were always rated higher than the anti-flirt version. In addition, the experiment revealed that the interactive version contributed to the user's enjoyment, increased their interest to continue the interaction or even to engage in a conversation with Alfred even though the differences were only significant for the interactive and the anti-flirt version (Hypothesis III). The users did not have the feeling that the agent was significantly more responsive in the interactive than in the non-interactive versions. The result is in conflict with a result we obtained for an earlier experiment with an eye-gaze controlled agent. In the earlier experiment, the interactive agent was perceived as more responsive than the non-interactive agent. The subjects felt, however, also more disturbed by the perceptive agent Eichner et al., 2007. Obviously, the users enjoyed the interactive version more and found it more engaging without perceiving it, however, as more interactive. The higher level of engagement was also reflected by the users' behavior. We were seeking more often for eye contact with the agent 
than in the non-interactive versions. Furthermore, the subjects found the interactive agent more realistic and indicated that it was more natural to interact with it.

\section{Conclusion}

In this paper, we presented an eye-gaze based interaction model for embodied conversational agents which incorporates studies of flirting in human-human interaction. The approach was tested using a 3D character that enables realistic gaze behaviors in combination with expressive mimics. We successfully integrated an eye tracker in a life-size application with a quite huge interaction screen display. The contact-free eye tracker has proven appropriate and reliable for a life-size interactive setting with a large interaction screen display. It was not perceived as intrusive or disturbing and thus the users could interact in a relatively natural manner. As we did not give the subjects special instructions how they should behave in front of the eye tracker, they moved freely without taking care of the eye tracker. The usage of such eye tracker is promising, as only 2 of 16 subjects had to be removed from the recordings as the eye tracker did not work trouble-free with them.

To enable smooth interactions, a high amount of alignment and coordination was required. In particular, the agent had to sense and respond to the user's gaze behavior in real-time. Despite of the technical challenges involved in this task, the interactive version was perceived as more natural than the non-interactive versions. The user's also had the impression that the agent had an interest in them without perceiving it as obtrusive. Both subjective user ratings as well as objective user observations revealed that the users were more eager to continue interaction with the agent.

Usually, attractiveness is considered as a prerequisite for successful flirting. Our subjects rated Alfred as sympathetic, but also little attractive. Nevertheless, the incorporation of flirting tactics has proven beneficicial. Thus, this can be taken as evidence that the flirting tactics as implemened in this work are of benefit to a much broader range of situations with agents than just dating, e.g. initiate human-agent interaction or regulating turn-taking in dialogues.

\section{Acknowledgements}

This work has been funded in part by the European Commission under grant agreement IRIS (FP7-ICT-231824).

\section{References}

Argyle and Cook, 1976. Argyle, Cook: Gaze \& Mutual Gaze. Cambridge University Press, Cambridge (1976)

Augsburg University, 2008. Augsburg University, Horde3D GameEngine (2008),

http://mm-werkstatt. informatik. uni-augsburg. de/projects/GameEngine/ 
Bee et al., 2009. Bee, N., Falk, B., André, E.: Simplified facial animation control utilizing novel input devices: A comparative study. In: International Conference on Intelligent User Interfaces (IUI 2009), pp. 197-206 (2009)

Bossi, 1995. Bossi, J.: Augen-Blicke. Zur Psychologie des Flirts, Huber, Bern (1995)

Cassell and Bickmore, 2003. Cassell, J., Bickmore, T.W.: Negotiated collusion: Modeling social languageand its relationship effects in intelligent agents. User Model. User-Adapt. Interact. 13(1-2), 89-132 (2003)

Cohen, 1992. Cohen, D.: Body Language in Relationships. Sheldon Press (1992)

Eichner et al., 2007. Eichner, T., Prendinger, H., André, E., Ishizuka, M.: Attentive presentation agents. In: Intelligent Virtual Agents (IVA 2007), pp. 283-295 (2007)

Ekman and Friesen, 1975. Ekman, P., Friesen, W.: Unmasking the Face. Prentice Hall, Englewood Cliffs (1975)

Facial Expression Repertoire, 2008. Facial Expression Repertoire, Filmakademie Baden-Württemberg (2008), http://research.animationsinstitut.de/

Givens, 1978. Givens, D.B.: The nonverbal basis of attraction: flirtation, courtship, and seduction. Psychiatry 41(4), 346-359 (1978)

Gratch et al., 2006. Gratch, J., Okhmatovskaia, A., Lamothe, F., Marsella, S., Morales, M., van der Werf, R., Morency, L.P.: Virtual rapport. In: Gratch, J., Young, M., Aylett, R.S., Ballin, D., Olivier, P. (eds.) IVA 2006. LNCS (LNAI), vol. 4133, pp. 14-27. Springer, Heidelberg (2006)

Hall, 1963. Hall, E.T.: A system for notation of proxemic behavior. American Anthropologist 65, 1003-1026 (1963)

Kleinke, 1986. Kleinke, C.L.: Gaze and eye contact: A research review. Psychological Bulletin 100(1), 78-100 (1986)

Pan and Slater, 2007. Pan, X., Slater, M.: A preliminary study of shy males interacting with a virtual female. In: PRESENCE 2007: The 10th Annual International Workshop on Presence (2007)

Sidner et al., 2004. Sidner, C.L., Kidd, C.D., Lee, C., Lesh, N.: Where to look: a study of human-robot engagement. In: IUI 2004: Proceedings of the 9th international conference on Intelligent user interfaces, pp. 78-84. ACM Press, New York (2004)

SMI, 2008. SMI. SMI iView X RED (2008),

http://www.smivision.com/en/eye-gaze-tracking-systems/products/ iview- $\mathrm{x}-\%$ red. html

Starker and Bolt, 1990. Starker, I., Bolt, R.A.: A gaze-responsive self-disclosing display. In: CHI 1990: Proceedings of the SIGCHI conference on Human factors in computing systems, pp. 3-10. ACM, New York (1990)

Tramitz, 1992. Tramitz, C.: Auf den ersten Blick. ADMOS Media GmbH (1992) 